\title{
Toward Self-Powered Internet of Underwater Things Devices
}

\author{
Jose Ilton de Oliveira Filho, Student Member, IEEE, Abderrahmen Trichili, Member, IEEE, Boon S. Ooi, Senior \\ Member, IEEE Mohamed-Slim Alouini, Fellow, IEEE, and Khaled Nabil Salama, Senior Member, IEEE
}

\begin{abstract}
Exploiting light beams to carry information and deliver power is suggested as a potential technology to recharge batteries in future generations of the Internet of Things (IoT) and Internet of Underwater Things (IoUT) devices while providing optical connectivity. Simultaneous lightwave information and power transfer (SLIPT) has been recently proposed as an efficient method of wireless power transfer between communicating terminals. In this article, we provide an overview of the various SLIPT techniques in time, power, and space domains. We also demonstrate two SLIPT scenarios through underwater channels, and we discuss the issues related to the hardware as well as system deployment in harsh environments.
\end{abstract}

Index Terms-SLIPT, energy harvesting, underwater optical wireless communication, Internet of Underwater Things

\section{INTRODUCTION}

W IRELESS power transfer has received considerable attention in recent years. Radio frequency (RF) simultaneous wireless information and power transfer (SWIPT) was proposed as a technique to transmit information and harvest energy by converting energy from an electromagnetic field into the electrical domain [1], [2]. SWIPT is considered a future generation energy transfer technology in wireless communication networks. However, beyond RF spectrum scarcity, RF energy harvesting suffers from relatively low efficiency and major technical problems related to the transmitting and receiving circuits [3]. Additional challenges are imposed by the electromagnetic safety and health concerns raised regarding high power RF applications (references are within [3]). SWIPT can equally be a source of interference to data transmission and RF pollution.

One alternative to the use of electromagnetic radiation for harvesting energy are the lightwaves emitted by lightemitting diodes (LEDs) and laser sources. Using light beams, it is possible to perform a transfer of energy and efficiently deliver data streams simultaneously. Simultaneous lightwave information and power transfer (SLIPT) can provide significant performance over RF-based SWIPT, leveraging the recent progress in license-free optical wireless technology [4]. Optical wireless power transfer (OWPT) can also be a complementary technology to visible light communication (VLC), as proposed in [4]-[6]. In the fundamental work of Fakidis et al. [4], the concept of indoor OWPT to small cells during darkness hours was investigated. Using visible lasers, the authors reported the successful transfer of $7.2 \mathrm{~W}$ of optical power over $30 \mathrm{~m}$. Rakia et al. designed a dual-hop VLC/RF

Authors are with the Computer, Electrical and Mathematical Sciences \& Engineering in King Abdullah University of Science and Technology, communication and energy harvesting scheme [5]. The relay harvests energy from the received optical signals transmitted over the first VLC hop, then uses the harvested energy to forward the received signals to a mobile terminal over the second RF hop. Furthermore, Diamantoulakis et al. proposed new strategies to optimize and enhance efficiency between the communication and energy harvesting functions for indoor applications with visible and infrared wireless communications [6].

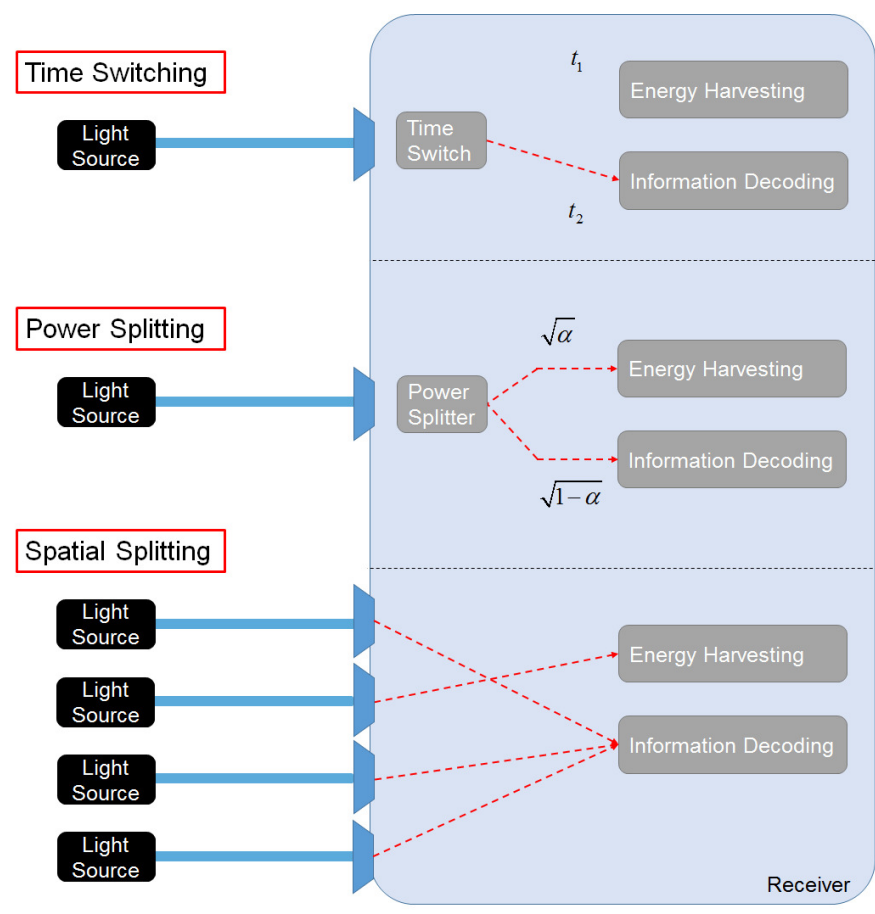

Fig. 1. Different SLIPT techniques: time switching, power splitting, and spatial splitting.

Motivated by the low cost of optical communication and energy harvesting circuit components over their RF counterparts, SLIPT can be an equally cost-effective solution for energy-constrained wireless systems, including remote sensors and autonomous self-powered devices. SLIPT is also very promising for applications in RF-sensitive environments and applications, such as medicine, smart houses, and aerospace [7].

Another potential use of SLIPT is powering Internet of Underwater Things (IoUT) devices. This application is motivated by the high demand for underwater communication 


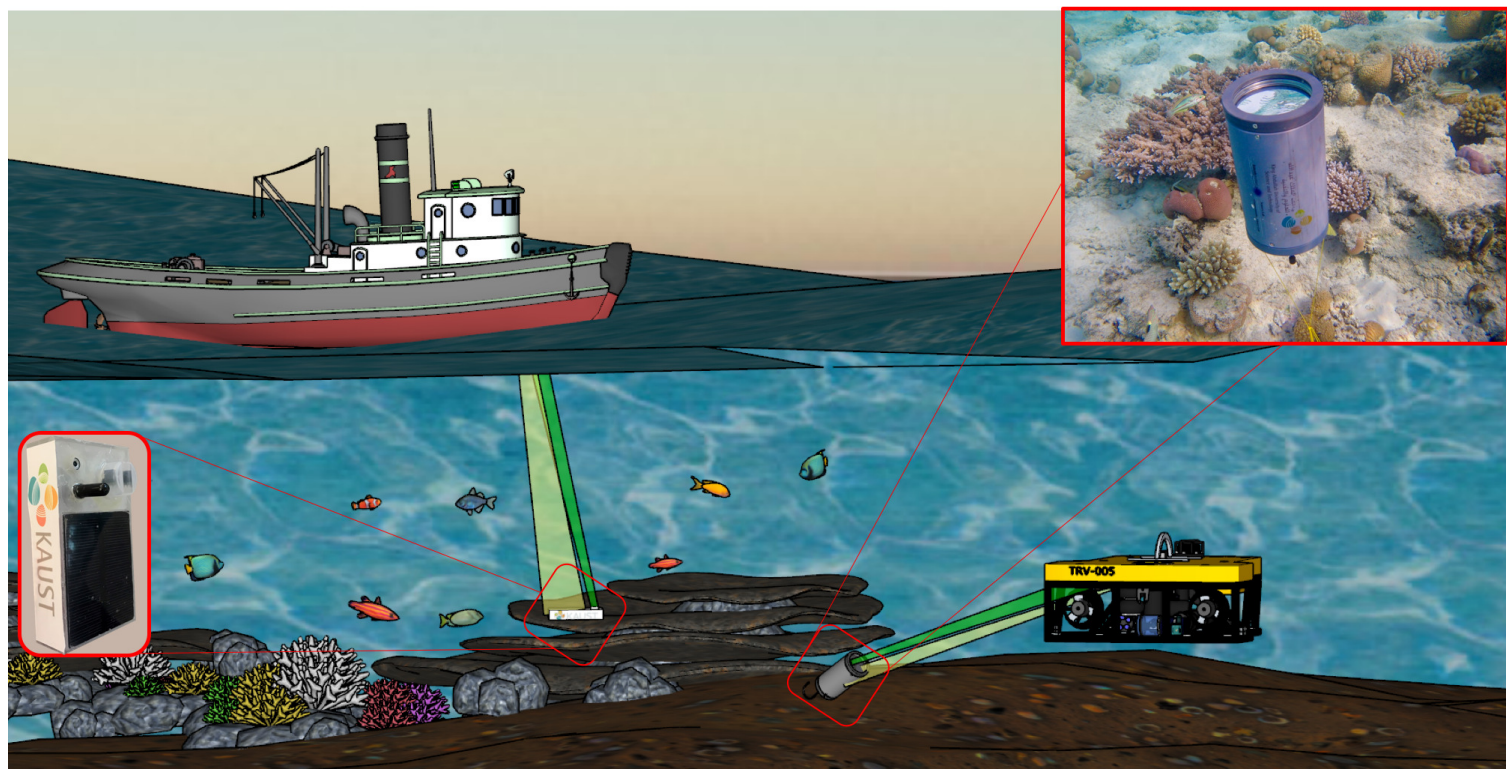

Fig. 2. Illustration of the use of self-powered IoUT devices in an underwater environment. The image of the IoUT in the right-hand-side of the schematic corresponds to a deployed self-powered device in the Red Sea (location is known as Abu Gisha island).

systems due to the continuous growth of underwater human activities, such as marine life protection, pollution tracking, marine current power grid connections, scientific data collection, undersea earthquakes monitoring, and offshore oil field exploration.

We note that wireless transmission underwater can be executed through acoustic, RF, and optical waves. Traditionally, acoustic communication has been used for underwater applications for many decades and can reach long propagation distances of several kilometers. The typical frequency range associated with acoustic communication spans $10 \mathrm{~Hz}$ to $1 \mathrm{MHz}$. However, this technology suffers from minimal available bandwidth and large latencies caused by the low propagation speed. Nevertheless, acoustic devices are prohibitively expensive and consume a high amount of energy. Underwater RF communication also requires costly antennas operating with high transmission powers. It should also be noted that RF waves are subject to high attenuation in seawater and can only reach considerable distances at the super-low frequency band of $30-300 \mathrm{~Hz}$ in shallow waters. These constraints make RF unappealing for most practical underwater communication purposes.

Compared to acoustic and RF communications, underwater optical wireless communication (UWOC) through ocean water can cover a broad frequency range and involve transferring a high amount of data such as several Gbit/s in an energyefficient manner. SLIPT, as a complementary technology to UWOC, can provide continuous connectivity and wireless power for devices and sensors in difficult-to-access locations. A significant additional advantage of this technique is the low cost of transmitters and receivers compared to RF and acoustic communication terminals. Several commercially available optical sources and detectors also have small foot-prints and can easily be integrated into IoUT modules.

In this paper, we present the different concepts of optical SWIPT or SLIPT. We then provide experimental demonstra- tions of time-switching SLIPT in an underwater environment for IoUT applications. We further discuss the open problems and propose key solutions for the deployment of marine SLIPT-based devices.

\section{SLIPT System DESIGN AND CONCEPT}

A SLIPT system requires a light source able to emit modulated signals and an optical receiver to decode information and harvest energy. Different possible techniques in various domains, including time, power, and space, can be harnessed to transmit information and harvest energy. The main differences between the three SLIPT techniques lie in the number of light sources and the receiver architecture, as shown in Fig. 1

\section{A. Time Switching}

In a time-switching configuration, the receiver, which is possibly an off-the-shelf solar cell (SC), switches between the energy harvesting and information decoding modes, better known as the photovoltaic and photoconductive modes, respectively. Both SLIPT functions are performed over two different time slots $t_{1}$ and $t_{2}$, as seen in Fig. 11. The quantity of harvested energy is ruled by the conversion efficiency of the SC. With the advances in the development of high-speed light sources, the maximum achievable transmission rate is mainly restricted by the bandwidth of the SC. Synchronizing the photovoltaic and photoconductive modes is crucial and can be done via hardware programming. A low-power relay can fulfill the switching function between the two SC modes.

\section{B. Power Splitting}

With a power-splitting approach, the light source continuously emits modulated signals. The receiving terminal is simultaneously charged while decoding the information carried by the incident light beam, as seen in Fig. 11. A key device 


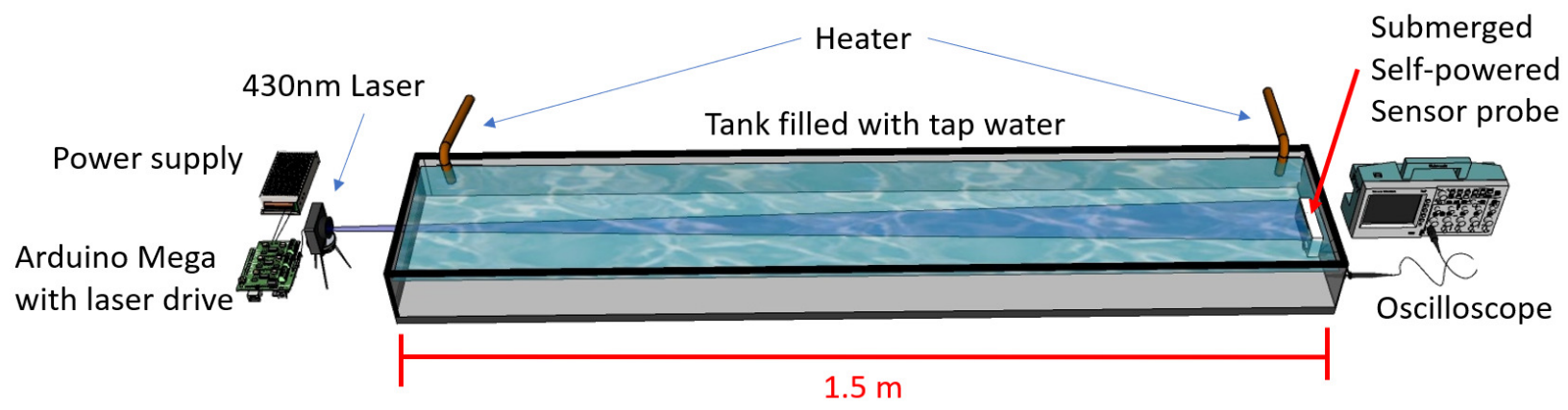

Fig. 3. Schematic of the experimental setup of time switching underwater SLIPT.

needed for this configuration is the power splitter, which splits the incident power into $\alpha$ and $(1-\alpha)$ quantities. The $\alpha P_{R}$ power portion is used to harvest energy, while the $(1-\alpha) P_{R}$ portion is used to decode the received signal. The powersplitting component can be a passive beam splitter (BS), which splits the incident beam from the light source into two beams (or possibly more). The BS can split the beam power into (un)evenly distributed portions. The power splitter can also be a component with variable splitting ratios, potentially increasing the system complexity. Different from the timeswitching approach, simultaneous energy and power transfer are fulfilled when using a power-splitting system. It is also possible to achieve higher transmission rates because the decoding can be performed via a high-speed photodiode (PD).

\section{Spatial Splitting}

The spatial-splitting approach is applied to a configuration involving multiple transmitters and multiple receivers with information decoding and energy harvesting capabilities, as depicted in Fig. 11 Each transmitter can transfer data or energy, and each receiver can harvest energy from multiple transmitters. Time switching can be applied within this configuration where the same receiver can act as an "energy harvester" and "signal decoder" over different time periods.

In the following section, we report two demonstrations of timeswitching SLIPT.

\section{DEMONSTRATIONS}

Multiple experimental SLIPT-related demonstrations, in free space and underwater media, have been reported in the literature. One of the first experimental reports demonstrated the use of a low-cost silicon SC to decode VLC signals emitted from an LED source at a rate of $3 \mathrm{kbit} / \mathrm{s}$ while harvesting energy from sunlight [8]. Wang et al. proposed a novel design for an optical receiver based on a multi-crystalline-silicon solar panel available on the market [9]. The authors reported a data rate of $11.84 \mathrm{Mbit} / \mathrm{s}$ and $2 \mathrm{~mW}$ of generated power over less than 1 $\mathrm{m}$ in an indoor environment. Zhang and co-workers achieved a data rate of $34.2 \mathrm{Mbit} / \mathrm{s}$ using an $8 \mathrm{~mm}^{2}$ organic SC as a VLC receiver [10]. Earlier demonstrations also involved the use of a $5 \mathrm{~cm}^{2}$ solar panel as a receiver for an underwater communication link over a 7-m long water tank [11]. A single gallium arsenide (GaAs) SC with a 3-dB bandwidth of 24.5
$\mathrm{MHz}$ was also used to perform a $0.5 \mathrm{~Gb} / \mathrm{s}$ transmission over a 2-m-long free-space link [12].

Here, we demonstrate two communication and energy harvesting scenarios through underwater media using two homemade IoUT devices. The IoUT devices can potentially be charged through light beams that can be emitted from a source fixed on a boat or an autonomous underwater vehicle, as depicted in Fig. 2 In the first experiment, we charge the battery of a submerged module equipped with a temperature sensor. We then transmit commands using a laser to the module. The temperature sensor is used to monitor the variable temperature of a water tank. In the second demonstration, we charge the capacitor of an IoUT device equipped with a camera and a low-power laser for real-time video streaming.

\section{A. Self-Powered Sensor Module}

The experimental setup of the first demonstration is depicted in Fig. 3. The transmitter was placed outside of a $1.5-\mathrm{m}$ long water tank and composed of an Arduino mega with a laser drive connected to a $430 \mathrm{~nm}$ laser diode. The receiver installed inside the water tank was formed by a low-cost monocrystalline silicon solar panel connected to a low-power latching switch controlled by a programmable system-on-chip (PSoC). The $55 \times 70 \mathrm{~mm}$ solar panel has a $3-\mathrm{dB}$ bandwidth of $100 \mathrm{kHz}$ in the photoconductive mode, costing 2 USD. The utilized switching mechanism allowed the solar panel to operate in either the photovoltaic or photoconductive mode at a given moment. In the photovoltaic mode, the photocurrent flows from the SC to a supercapacitor to store the harvested energy. The flow can be stopped, and the solar panel becomes reverse-biased, thereby switching to the photoconductive mode. This dual-mode results in a significant advantage for energy harvesting and information decoding.

Moreover, in our circuit design, we considered the maximum power point tracking (MPPT) technique for the solar panel, which generally maximizes the extracted power in the photovoltaic mode, as the amount of received power from light varies in an underwater environment. The MPPT of the solar panel in our circuit worked without affecting the decoded signals, as both SC modes were independent.

The module awakes upon receiving a light beam on the solar panel. The battery voltage $V_{B}$ is then measured. If $V_{B}<V_{t h}$, where $V_{t h}$ for our module is a threshold voltage 
of $3.6 \mathrm{~V}$, the sensor begins measuring the temperature. The collected data is saved on a secure digital (SD) memory card connected to the device, which then enters a sleep mode. If $V_{B} \geq V_{t h}$, the solar panel switches to a photoconductive mode and receives commands, including switching the sensor "on" or "off", and sending and re-transmitting the saved data on the memory card using an integrated red laser. Upon executing all the needed commands, the solar panel switches to energy harvesting mode. Upon reaching a full battery charge, the module enters sleep mode.

A full charge of the $840-\mathrm{mW}$ module battery required approximately 124 minutes using the blue laser. The reached throughput when the solar panel acted as an information receiver was equal to $500 \mathrm{kbit} / \mathrm{s}$. The temperature sensor was then activated to measure the thermal changes inside the water tank over a time window of more than two hours. The thermal variation of the water was controlled using two chillers fixed on the sides of the tank. The temperature evolution of the water as a function of time is shown in Fig. 4.

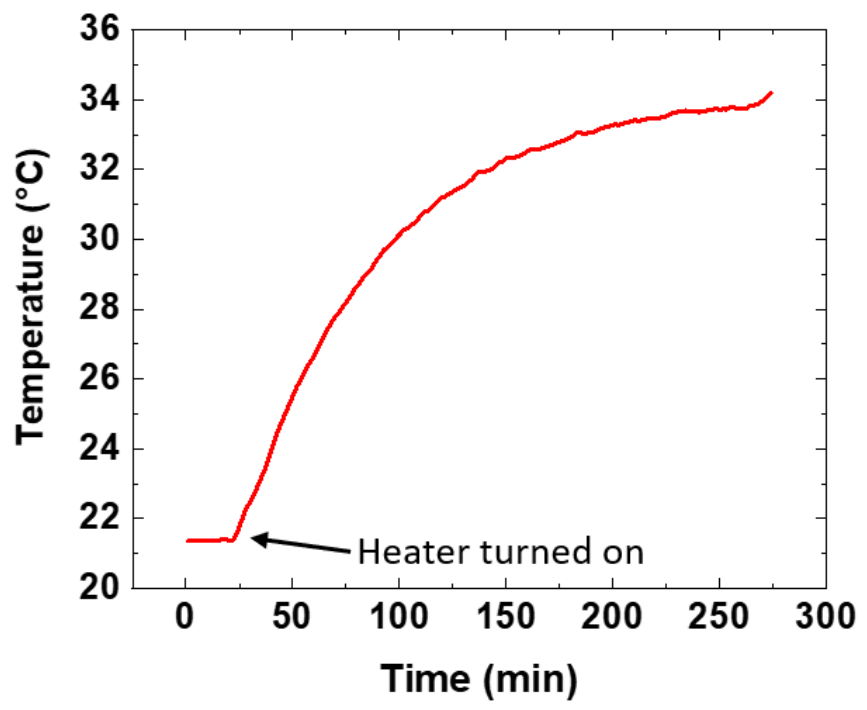

Fig. 4. Temperature evolution in the water tank as a function of time.

\section{B. Self-Powered Underwater Camera}

The second demonstration involved an IoUT device equipped with an analog camera with a frame rate of 30 fps for underwater live video streaming. The IoUT is formed from an analog front-end PSoC circuit, powered by a $5 \mathrm{~F}$ supercapacitor, charged via a solar panel like the one utilized in the first demonstration. A low-power red laser was also connected to the device circuit for video transmission. The device was fixed at the bottom of a tank filled with seawater, as shown in Fig. 5. The water inside the tank was changed dynamically using a pump and pipes directly connected to the Red Sea. First, a 4.8 W LED source was fixed $30 \mathrm{~cm}$ away from the receiver. The light source was partially submerged and powered externally. At a distance of $30 \mathrm{~cm}$, the LED power measured with a lux meter was 1325 lux. The full supercapacitor charge required approximately $1 \mathrm{~h} 30 \mathrm{~min}$. Once fully charged, the device was used to establish 1-minute-long real-time streaming of a video captured by the analog camera.

The main challenge for the real-life deployment of such a self-powered device would be strong water motion significantly shaking the device. The use of a large-area solar panel to harvest energy and decode information can ease the strict pointing requirements when compared to state-of-theart UWOC systems based on limited active area detectors. However, water motion can significantly affect the pointing of the device's laser towards any detector. In the next section, we further detail the different problems that self-powered devices encounter.

\section{OPEN PROBLEMS}

There are different challenges associated with the various SLIPT techniques. Challenges are either related to the hardware such as the bandwidth of the solar cell and the battery lifetime, or the propagation effects over wireless underwater media. Additional challenges are imposed when moving from a point-to-point system to a network level. In this section, we discuss the open problems of the SLIPT technology and propose future research directions to cope with deployment challenges.

\section{A. Hardware Challenges}

One of the main challenges for SLIPT is increasing the transmission rate of optical communication links. This problem stems from the bandwidth of the receiving terminal, which is limited by the decoding bandwidth of the SC, mainly for time-switching SLIPT configurations. The 3-dB bandwidth of commercially available silicon SCs is usually restricted to tens of $\mathrm{kHz}$. Using advanced modulation formats such as M-quadrature amplitude modulation-orthogonal frequency division multiplexing (M-QAM OFDM) can significantly scale the transmission capacity. However, implementing such a modulation scheme may result in complexity in the transmitting and receiving circuits. High order modulation formats also have an impact on the power consumption at the receiver end. However, using higher 3-dB bandwidth III-V SCs, such as the GaAs cell used in [12], can further increase the transmitted data rate.

Limitations on data transmission rates can also be alleviated in the power-splitting SLIPT configuration. The idea is to use a high-bandwidth PD to decode information signals instead of using solar cells at the expense of pointing errors. We note that the detection areas of commercially available high-bandwidth detectors are limited to only a few tens of $\mathrm{mm}^{2}$, due to the limit imposed by the resistor-capacitor time constant [13]. This limitation results in a strict angle of view that requires maintaining a perfect system alignment. Using high-speed PDs can also generate additional complexity for the power splitter, which should be adapted to deliver enough power to decode the signals.

Further challenges are related to the battery lifetimes of IoUT devices. Battery lifetime is dependent on the enabled features offered by each device; for example, the downlink data rate 


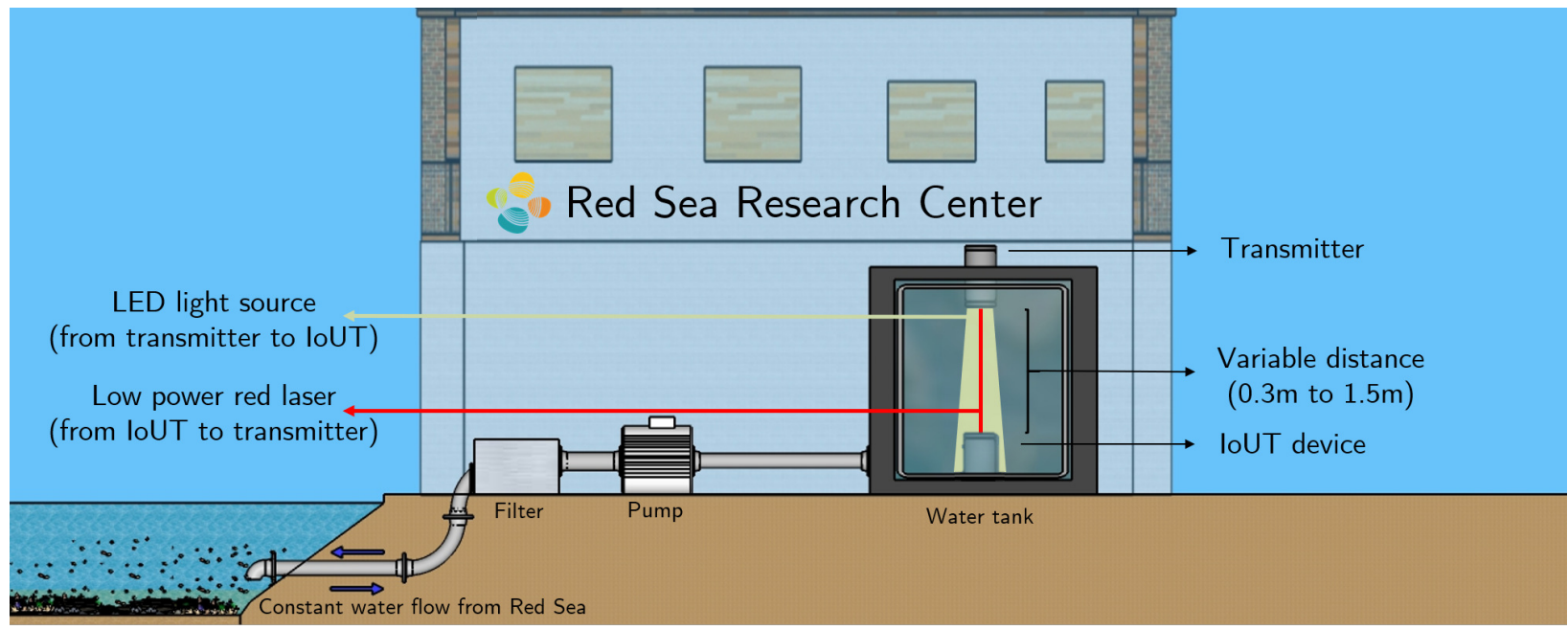

Fig. 5. Schematic illustration of the underwater SLIPT demonstration. Charging the module is performed using an LED source. The device is streaming a real-time captured video using a red color laser.

TABLE I

CURRENT CONSUMPTION FOR DIFFERENT ENABLED FEATURES

\begin{tabular}{|c|c|c|c|}
\hline Source & $\begin{array}{c}\text { Current } \\
\text { Consumption }\end{array}$ & $\begin{array}{c}\text { Downlink } \\
\text { Throughput }\end{array}$ & Enabled Features \\
\hline $3.7 \mathrm{~V}$ & $102 \mathrm{~mA}$ & $500 \mathrm{kbit} / \mathrm{s}$ & Wi-Fi, Bluetooth \\
\hline $3.7 \mathrm{~V}$ & $36 \mathrm{~mA}$ & $500 \mathrm{kbit} / \mathrm{s}$ & System with clock at $10 \mathrm{MHz}$ \\
\hline $3.7 \mathrm{~V}$ & $11 \mathrm{~mA}$ & $115.2 \mathrm{kbit} / \mathrm{s}$ & System with clock at $3 \mathrm{MHz}$ \\
\hline $3.7 \mathrm{~V}$ & $7 \mathrm{~mA}$ & N/A & Sensing and saving data \\
\hline $5 \mathrm{~V}$ & $110 \mathrm{~mA}$ & N/A & Video streaming \\
\hline $5 \mathrm{~V}$ & $236 \mathrm{~mA}$ & $500 \mathrm{kbit} / \mathrm{s}$ & $\begin{array}{c}\text { Video streaming, } \\
\text { Wi-Fi and Bluetooth }\end{array}$ \\
\hline
\end{tabular}

affects the energy consumption level of an IoUT device significantly. To assess the impact of various features on energy consumption levels, we collected the current consumption of a fully awake module powered by different sources (Table I). The module can perform various functions, including sensing, video streaming, and information decoding. In some cases, the module decodes signals at different data rates. We notated the cases when the module was not decoding any signals with N/A in the table. If no signal is being decoded, sensing, and saving data operations put the less burden on the energy source. However, applications such as video streaming are powerhungry.

Equipping the devices with $\mathrm{Wi}-\mathrm{Fi}$ and Bluetooth is useful for mobile IoUTs. These two technologies can be used to communicate with nearby floating devices fixed on boats or buoys. Using Wi-Fi and Bluetooth at sea surfaces helps to avoid the pointing errors that result in strong underwater currents or waves, and the solar detector also harvests energy from ambient light in the daytime.

\section{B. Underwater Channel Propagation Effects}

When propagating through the water, the intensity of a light beam decays exponentially along the propagation direction $z$, from the initial intensity $I_{0}$ following the Beer-Lambert law expressed as $I=I_{0} \exp (-a z)$, with $a$ being the attenuation coefficient. As such, $a$ is obtained by summing the contribution of two main phenomena, $b$ and $c$, the absorption and scattering coefficients, respectively. Beams propagating through the water are also subject to turbulence, which is due to random temperature in-homogeneity, salinity variations, or air-bubbles. Temperature fluctuations and salinity variations result in rapid changes in the refractive index of the water, leading to signal intensity fluctuations. Depending on their sizes and populations, air-bubbles through the propagation path can partially or wholly block light beams, therefore introducing severe signal fading. The existing literature includes statistical models to estimate the impact of a wide range of underwater turbulence occurrences [14]. When establishing a link to transfer power and information over an underwater channel, attenuation and turbulence effects should both be considered, as the goal is to ensure the delivery of enough power for the two SLIPT functions.

Relaying schemes can also suppress the impact of attenuation and extend the communication ranges of IoUT devices. Cooperative diversity is a fundamental key to moving from a point-to-point configuration to an IoUT network setting, and using relay nodes can increase overall performance in communication and energy harvesting. One or multiple neighboring nodes can extend the battery lifetime of a device. Furthermore, a network setting may be an efficient solution against the effect of turbulence. With a network of IoUT nodes, information transmission can occur over multiple paths.

Another viable way to reduce the impact of turbulence is to use multiple wavelengths for the information transfer and energy harvesting functions. Employing multiple wavelengths can provide a diversity gain over the harsh underwater environment if different copies of the same signal are encoded over distinct carrier wavelengths, which are affected differently by turbulence-induced distortions. For example, light attenuation in clear seawater is minimum in the blue-green region, while longer wavelengths are more effective in mitigating the effect 
of turbulence. In clear water, the use of multiple wavelengths to carry independent data streams can considerably scale the transmission capacity.

Using the example of a two-wavelength system, one of the wavelengths can transfer energy while the other carries the data streams, ensuring the continuous connectivity of the device. Such a scheme can be seen in wavelength-based power-splitting SLIPT, where the power-splitting component that we previously described in section II-B can be simply a wavelength filter dichroic mirror, for example. The two wavelengths can also act as independent data carriers while charging the same device simultaneously. In this configuration, the device should have the capability to separate the two wavelengths. Then, for each wavelength, the power is split into two portions, the first for information decoding, and the second handles energy harvesting.

\section{Beam Divergence}

While propagating through an unguided medium, a light beam tends to diverge, leading to an increase of the radius. Losses due to beam divergence can be denoted as geometrical attenuation, which scales with the propagation distance. Beam divergence is mainly related to the light source used at the transmitter, as well as the collimation system, if one is used. Beam divergence is also proportional to the source wavelength. Taking into account beam divergence is crucial for SLIPT systems. The power portion at the receiver should fulfill the two SLIPT functions. For short distances of a few meters, LED sources can deliver energy and information signals. However, for longer lengths, laser sources should be utilized, as they diverge much less than LED sources.

\section{Path Obstructions}

Obstructed propagation paths are another limiting factor for underwater SLIPT. Obstructions may be overcome using the enhanced scattering of ultraviolet (UV) light, which is harnessed to establish non-line of sight (NLoS) connections. Nonetheless, this approach requires using solar-blind photovoltaic cells to harvest energy from the UV light. This technique should be carefully studied to avoid any health issues related to UV exposure.

Cooperative relaying between devices may also address path obstructions.

\section{E. Network Architecture Challenges}

While providing several benefits, establishing an IoUT network architecture is challenging. The first issue is developing the proper routing and scheduling techniques for a network of self-powered devices [15]. Routing and scheduling protocols should provide a compromise between energy efficiency and latency. The second issue is the dependence of the network lifetime on the nodes' batteries. Equipping some of the network nodes with additional energy harvesting capability would then prolong the network lifetime. Another solution is to harvest energy from microbial fuel cells (references are within [15]). Furthermore, as GPS cannot operate underwater, the exact locations of the network's nodes are challenging to find with minimum energy consumption, which must be investigated [15].

\section{CONCLUSION}

Throughout this article, we provided an overview of SLIPT, a key technology in green energy transfer that could exploit different degrees of freedom, including time, power, and space. Moreover, we presented two underwater experimental demonstrations of time switching SLIPT. In our first proof-of-concept test, we charged the battery of a submerged module using a blue laser. Then, we successfully transmitted commands at a rate of $500 \mathrm{kbit} / \mathrm{s}$ through a $1.5 \mathrm{~m}$ underwater link. We also collected data using a self-powered temperature sensor. In the second experiment, we transmitted commands and charged the capacitor of a device equipped with a low-power red laser and analog-camera. Beyond the experimental demonstrations, the open problems, and design considerations of building underwater SLIPT systems were studied. As SLIPT is still a largely unexplored field, it requires more in-depth research efforts to solve its several technical issues and channelrelated challenges before the wide-scale deployment of this technology.

\section{ACKNOWLEDGMENT}

This work was supported by funding from King Abdullah University of Science and Technology. The authors would like to thank the Red Sea Research Center and Coastal \& Marine Resources Core Lab (CMOR) for helping with the testing and deployment of the prototypes.

\section{REFERENCES}

[1] I. Krikidis, S. Timotheou, S. Nikolaou, G. Zheng, D. W. K. Ng, and R. Schober, "Simultaneous wireless information and power transfer in modern communication systems," IEEE Commun. Mag., vol. 52, no. 11, pp. 104-110, Nov. 2014

[2] X. Lu, P. Wang, D. Niyato, D. I. Kim, and Z. Han, "Wireless networks with RF energy harvesting: A contemporary survey," IEEE Commun. Surv. Tutor, vol. 17, no. 2, pp. 757-789, 2nd Quart. 2015.

[3] T. D. Ponnimbaduge Perera, D. N. K. Jayakody, S. K. Sharma, S Chatzinotas, and J. Li, "Simultaneous wireless information and power transfer (SWIPT): Recent advances and future challenges," IEEE Commun. Surv. Tutor., vol. 20, no. 1, pp. 264-302, 1st Quart. 2018.

[4] J. Fakidis, S. Videv, S. Kucera, H. Claussen, and H. Haas, "Indoor Optical Wireless Power Transfer to Small Cells at Nighttime," J. Lightwave Technol., vol. 34, no. 13, pp. 3236-3258, Jul. 2016.

[5] T. Rakia, H. Yang, F. Gebali, and M.-S. Alouini, "Optimal design of dual-hop VLC/RF communication system with energy harvesting," IEEE Commun. Lett., vol. 20, no. 10, pp. 1979-1982, Oct. 2016.

[6] P. D. Diamantoulakis, G. K. Karagiannidis, and Z. Ding, "Simultaneous lightwave information and power transfer (SLIPT)," IEEE Trans. Green Commun. NetW., vol. 2, no. 3, pp. 764-773, Sep. 2018.

[7] G. Pan, P. D. Diamantoulakis, Z. Ma, Z. Ding and G. K. Karagiannidis, "Simultaneous lightwave information and power transfer: Policies, techniques, and future directions," IEEE Access, vol. 7, pp. 28250-28257, 2019.

[8] S.-M. Kim, and J.-S. Won, "Simultaneous reception of visible light communication and optical energy using a solar cell receiver," 2013 International Conference on ICT Convergence (ICTC), Jeju, 2013, pp. 896-897.

[9] Z. Wang, D. Tsonev, S. Videv, and H. Haas, "On the design of a solarpanel receiver for optical wireless communications with simultaneous energy harvesting," IEEE J. Sel. Areas Commun., vol. 33, no. 8, pp. 1612 1623, Aug. 2015. 
[10] S. Zhang, D. Tsonev, S. Videv, S. Ghosh, G. A. Turnbull, I. D. W. Samuel, and H. Haas, "Organic solar cells as high-speed data detectors for visible light communication," Optica, vol. 2, pp. 607-610, 2015.

[11] M. Kong, B. Sun, R. Sarwar, J. Shen, Y. Chen, F. Qu, J. Han, J. Chen, H. Qin, and J. Xu, "Underwater wireless optical communication using a lens-free solar panel receiver," Opt. Commun., vol. 426, pp. 94-98, 2018.

[12] J. Fakidis, S. Videv, H. Helmers and H. Haas, "0.5-Gb/s OFDM-based laser data and power transfer using a GaAs photovoltaic cell," IEEE Photon. Technol. Lett., vol. 30, no. 9, pp. 841-844, May 2018.

[13] Z. P. Zhao, J. G. Liu, Y. Liu, N. H. Zhu, "High-speed photodetectors in optical communication system," J. Semicond., vol. 38, 121001, 2017.

[14] E. Zedini, H. M. Oubei, A. Kammoun, M. Hamdi, B. S. Ooi, and M.-S. Alouini, "Unified statistical channel model for turbulence-induced fading in underwater wireless optical communication systems," IEEE Trans. Commun., vol. 67, no. 4, pp. 2893-2907, Apr. 2019.

[15] N. Saeed, A. Celik, T. Y. Al-Naffouri, M.-S. Alouini, "Underwater optical wireless communications, networking, and localization: A survey," Ad Hoc Networks, vol. 94, 101935, 2019.

Jose Ilton de O. Filho received the B.S. degree in Electrical Engineering from the Federal University of Piaui, Brazil, in 2017. He is currently pursuing a Ph.D. degree in Electro-Physics at King Abdullah University of Science and Technology (KAUST), Thuwal, Makkah Province, Saudi Arabia. His current areas of interest include instrumentation, sensing, optical communication, and wireless energy harvesting.

Abderrahmen Trichili received his diplôme d'ingénieur and Ph.D. degree in Information and Communication Technology from l'École Supérieur des Communications de Tunis (SUP'COM, Tunisia) in 2013 and 2017, respectively. He is currently a Postdoctoral Fellow in CEMSE at KAUST. His current areas of interest include space division multiplexing, orbital angular momentum multiplexing, free-space optical communication, and underwater wireless optical communication systems.

Boon S. Ooi is a Professor of Electrical Engineering at KAUST. He received the Ph.D. degree from the University of Glasgow (UK) in 1994. He joined KAUST from Lehigh University (USA) in 2009. His recent research is concerned with the study of III-nitride-based materials and devices and lasers for applications such as solid-state lighting, visible-light and underwater wireless optical communications, and energy-harvesting devices. He has served on the technical program committee of CLEO, IPC, ISLC, and IEDM. He currently serves on the editorial board of Optics Express and IEEE Photonics Journal $\mathrm{He}$ is a Fellow of OSA, SPIE, and IoP (UK).

Mohamed-Slim Alouini received the Ph.D. degree in Electrical Engineering from the California Institute of Technology (Caltech), Pasadena, CA, USA, in 1998. He served as a faculty member in the University of Minnesota, Minneapolis, MN, USA, then in the Texas A\&M University at Qatar, Education City, Doha, Qatar before joining KAUST, Thuwal, Makkah Province, Saudi Arabia as a Professor of Electrical Engineering in 2009. His current research interests include the modeling, design, and performance analysis of wireless communication systems.
Khaled N. Salama received the B.S. degree from the Department Electronics and Communications, Cairo University, Cairo, Egypt, in 1997, and the M.S. and Ph.D. degrees from the Department of Electrical Engineering, Stanford University, Stanford, CA, USA, in 2000 and 2005, respectively. He was an Assistant Professor at Rensselaer Polytechnic Institute, NY, USA, between 2005 and 2009. He joined King Abdullah University of Science and Technology (KAUST) in January 2009, where he is now a professor, and was the founding Program Chair until August 2011. He is the director of the sensors initiative a consortium of 9 universities (KAUST, MIT, UCLA, GATECH, Brown University, Georgia Tech, TU Delft, Swansea University, the University of Regensburg and the Australian Institute of Marine Science (AIMS). His work on CMOS sensors for molecular detection has been funded by the National Institutes of Health (NIH) and the Defense Advanced Research Projects Agency (DARPA), awarded the Stanford-Berkeley Innovators Challenge Award in biological sciences and was acquired by Lumina Inc. He is the author of 250 papers and 18 US patents on low-power mixed-signal circuits for intelligent fully integrated sensors and neuromorphic circuits using memristor devices. 